\title{
Clinical course of nontraumatic, nonaneurysmal subarachnoid hemorrhage: a single-institution experience
}

\author{
Jobyna Whiting, M.D., ${ }^{1}$ John Reavey-Cantwell, M.D., ${ }^{1}$ Gregory Velat, M.D., ${ }^{1}$ \\ Gregory Fautheree, M.D., ${ }^{1}$ Christopher Firment, M.D., ${ }^{2}$ Stephen Lewis, M.D., ${ }^{1}$ \\ AND BRIAN HOH, M.D. ${ }^{1}$ \\ Departments of ${ }^{1}$ Neurosurgery and ${ }^{2}$ Radiology, University of Florida, Gainesville, Florida
}

\begin{abstract}
Object. Angiogram-negative subarachnoid hemorrhage (SAH) accounts for 15\% of nontraumatic SAH and has been reported with low morbidity and mortality rates. We report on a large series of patients with angiogram-negative $\mathrm{SAH}$ who experienced an atypical nonbenign clinical course.

Methods. Between December 2001 and November 2006, 95 patients with spontaneous nonaneurysmal SAH and negative initial angiographic evaluation were treated at the University of Florida. The authors retrospectively reviewed the patients' medical records and radiological images to determine associated morbidity and mortality.

Results. Aneurysms were found in 6 of the 95 patients on follow-up imaging after an initial negative angiogram (6.3\% false negative rate); these patients were excluded leaving 89 patients as the study group. Hydrocephalus necessitating temporary CSF diversion developed in 22 of these patients $(25 \%) ; 12$ (13\%) ultimately required permanent CSF diversion. Clinically significant vasospasm developed in 4 patients $(4 \%)$, and $2(2 \%)$ had cerebral infarctions. Three patients $(3 \%)$ died.

Conclusions. The authors' experience with a large series of angiogram-negative SAH patients who had an atypical nonbenign clinical course associated with hydrocephalus, vasospasm, stroke, and mortality differs significantly from previously published case series of angiogram-negative SAH. (DOI: 10.3171/2009.2.FOCUSO92)
\end{abstract}

\section{KEY WORDS • nonaneurysmal subarachnoid hemorrhage • vasospasm • morbidity - mortality}

$\mathrm{I}$ $\mathrm{N} \sim 15 \%$ of patients with spontaneous SAH, no obvious source of hemorrhage can be demonstrated on cerebral angiography. ${ }^{6,13,14,19-21}$ The clinical course of these patients has been reported in the past to be benign compared with patients with aneurysmal SAH. We, however, had not found this to be true in patients with NASAH (nonaneurysmal SAH) at our institution. We reviewed our experience with patients with nontraumatic NASAH to determine the rate of associated morbidity and mortality.

\section{Methods}

We conducted this study under an institutional review board-approved protocol. All data were obtained and maintained with the protection of patient privacy, and in accordance with the Health Insurance Portability and Accountability Act guidelines.

Inclusion criteria for the study included age $>18$ years, SAH documented by a noncontrast head CT or by

Abbreviations used in this paper: $\mathrm{NASAH}=$ nonaneurysmal $\mathrm{SAH} ; \mathrm{SAH}=$ subarachnoid hemorrhage. xanthochromic CSF, and a negative cerebral angiogram. Exclusion criteria for the study included trauma, age $<18$ years, and the presence of a cerebral aneurysm or other intracranial vascular abnormality.

All patients who underwent cerebral angiography for the diagnosis of SAH between December 2001 and November 2006 were identified. All medical records, CT scans, and cerebral angiograms were reviewed.

Ninety-five patients were initially included in the study. Six patients had aneurysms on follow-up imaging, giving a detection rate for second angiograms of $9.4 \%$ (6 of 64 imaging studies). The 6 patients found to have aneurysms on follow-up imaging were excluded from further review. Eighty-nine patients had no aneurysm or vascular abnormality ever identified, and their cases were used for the analysis.

\section{Results}

All patients underwent an initial diagnostic cerebral angiogram. Sixty-three of the 89 patients had at least 1 additional cerebral angiogram. Seven patients had 1 cerebral angiogram followed by CT angiography for addi- 
J. Whiting et al.

TABLE 1: Type of follow-up imaging performed after initial negative cerebral angiogram

\begin{tabular}{lc}
\hline \multicolumn{1}{c}{ Imaging } & No. of Patients \\
\hline repeat cerebral angiography & 63 \\
CT angiogram & 7 \\
no follow-up imaging* & 19 \\
\hline
\end{tabular}

* Three of the 19 patients with no follow-up imaging died prior to further testing. The remaining 16 were lost to follow-up.

tional imaging. Nineteen patients had no follow-up imaging after their initial diagnostic cerebral angiograms, with follow-up imaging defined as imaging performed a week or more after the initial imaging study. Three of these patients died before follow-up images could be obtained. One patient presented neurologically intact, 8 days after initial SAH. This patient underwent a cerebral angiogram on admission and was discharged home with no follow-up imaging. The remaining 15 patients have no documented follow-up from this institution, so additional imaging was unable to be determined. The methods of follow-up imaging are given in Table 1.

The median age of the 89 patients was 56 years (range $20-88$ years). Forty-four of the 89 patients were women and 45 patients were men.

Study patients were assigned a Fisher grade based on their presenting hemorrhage pattern on noncontrast head CT. The Fisher grade was 1 in 11 patients (12\%), 2 in 16 (18\%), 3 in 43 (48\%), and 4 in 5 (6\%) (Table 2). In 14 cases $(16 \%)$ the initial head CT was performed at another facility, and these studies were not available for review. The presenting Fisher grade could not be confirmed in these cases.

Three patients tested positive for cocaine and 1 tested positive for methamphetamine at the time of hemorrhage. Fourteen patients were known smokers at the time of hemorrhage.

Early hydrocephalus was determined by the need for ventriculostomy placement during the initial hospitalization. Early hydrocephalus, requiring temporary CSF diversion, developed in 22 patients (25\%).

Late or persistent hydrocephalus was determined by the need for permanent CSF diversion via the placement of a ventriculoperitoneal shunt. Twelve patients (13\%) had late or persistent hydrocephalus and required shunts. Of these 12 patients, 2 did not have diagnosed early hydrocephalus, and they were noted to have symptomatic ventricular enlargement during follow-up.

Vasospasm developed in 4 (4\%) of the 89 patients studied. Two of these patients had a presenting Fisher grade of 3, and 2 had unknown presenting Fisher grades. In 2 of the patients with vasospasm associated infarctions developed.

Three patients (3\%) died during their initial hospitalization. Two of these patients were moribund upon presentation, and their status never improved. One patient who arrived moribund had undergone prolonged resuscitation in the field and was assumed to have a severe associated anoxic brain injury. No posthumous examination
TABLE 2: Fisher grade on CT scans performed at presentation*

\begin{tabular}{cc}
\hline Fisher Grade & No. of Patients (\%) \\
\hline 1 & $11(12)$ \\
2 & $16(18)$ \\
3 & $43(48)$ \\
4 & $5(6)$ \\
unknown† & $14(16)$ \\
\hline
\end{tabular}

* Without administration of contrast medium.

$\dagger$ In 14 cases, the initial imaging studies were performed at another institution and were not available for review at the time of retrospective data collection.

was performed in either of these 2 cases. One patient was neurologically intact on presentation and then had a sudden cardiac arrest during a head CT on hospital Day 7. Autopsy revealed myocardial infarction. There was no evidence of intracranial vascular abnormality on autopsy. The Fisher grade at presentation was 3 in all of the patients who died. Two of the patients had early hydrocephalus, which was treated with placement of an external ventricular drain in both cases. Table 3 shows all the major complications noted.

Feeding tubes were placed in 7 patients $(7.9 \%)$ during their initial hospitalization. Five patients $(5.6 \%)$ required tracheostomy placement. Three patients (3.3\%) developed deep venous thromboses. Two patients $(2.2 \%)$ had myocardial infarctions during their hospitalizations. Two patients $(2.2 \%)$ had new-onset seizures. Table 4 shows all of the additional complications noted during our patients' initial hospitalizations.

\section{Discussion}

A significant rate of morbidity and mortality was found in this single-institution review of cases of NASAH. This is in contrast to most published data concerning NASAH, which shows benign outcomes and few morbidities. ${ }^{1-5,7,9,14-17,19,22}$

Previously reported rates of hydrocephalus in NASAH range from 0 to $15 \%$, which is markedly lower than the $26 \%$ rate found in our patients. ${ }^{4,5,8,10,15,16,22}$ This difference may actually be larger than our numbers suggest, as most of the previous studies report hydrocephalus based on increased ventricular size and do not remark on clinical status. We reported only clinically significant increases in ventricular size that were accompanied by symptoms of increased intracranial pressure. It is likely that this definition excludes many cases of asymptomatic ventricular enlargement that would have been included in previously published rates of hydrocephalus.

One reason for the difference in incidence of hydrocephalus may be the higher Fisher grade in our patients' CT scans: $48 \%$ of our patients presented with Fisher Grade 3 SAH, and 6\% with Fisher Grade 4 SAH. Previously reported rates of Fisher Grade 3 are infrequent in patients with NASAH, and range from 10 to $33 \%$. There are no previously reported cases of Fisher Grade 4 NASAH to our knowledge. ${ }^{8,10,12}$ 
Nontraumatic nonaneurysmal SAH

TABLE 3: Major complications during hospitalization

\begin{tabular}{lc}
\hline Complication & No. of Patients (\%) \\
\hline early hydrocephalus & $22(25)$ \\
late hydrocephalus & $12(13)$ \\
vasospasm & $4(4)$ \\
infarction & $2(2)$ \\
death & $3(3)$ \\
\hline
\end{tabular}

The incidence of vasospasm in previously reported series of NASAH ranges from 0 to $15 \%$. $^{1,2,4,5,7,10,11,14,16,18} \mathrm{In}$ 1983, Beguelin and Seiler ${ }^{2}$ described a group of 42 patients with NASAH. They reported 5 deaths in their patient group. All 5 of the patients who died were enrolled in the treatment arm of a study evaluating antifibrinolytic treatment as a potential means of vasospasm prophylaxis and received tranexamic acid daily for 3 weeks. The authors presumed that the deaths were secondary to ischemia from cerebrovascular spasm, although spasm was only documented angiographically in 1 patient. Because of the potentially confounding issue of treatment with tranexamic acid, these data cannot be considered representative of the natural history of vasospasm incidence in patients with NASAH.

A review of 143 patients with NASAH published by Jung et al. in $2006,{ }^{10}$ reported a vasospasm rate of $15 \%$, although this may have included asymptomatic vasospasm. In our series, $4 \%$ of patients developed symptomatic vasospasm.

The incidence of cerebral infarction was $8 \%$ in 92 patients with NASAH reported on by Duong et al. ${ }^{5}$ None of these cases appear to be related to cerebral vasospasm, and the etiology of the infarcts is unclear. The overall rate of cerebral infarction in our patient group was $2 \%$, and all cases were associated with the presence of clinically significant vasospasm that was refractory to endovascular treatment. In fact, in spite of a relatively low overall risk of cerebral infarction in our patients, $50 \%$ of those who experienced clinically significant vasospasm went on to have associated cerebral infarction despite maximal medical and endovascular therapy.

The mortality rate in previously reported series of NASAH ranges from 0 to $12 \%$. Beguelin and Seiler ${ }^{2}$ reported that 5 of 42 patients treated with tranexamic acid for vasospasm prophylaxis died. Jain et al. ${ }^{9}$ reported a $2.3 \%$ mortality rate in a series of 43 patients with NA$\mathrm{SAH}$, and Duong et al. ${ }^{5}$ reported a $2.2 \%$ mortality rate in their 1996 article on their 92-patient series. In other case series involving patients with NASAH a mortality rate of $0 \%$ has been reported. Our study demonstrated a mortality rate of $3 \%$, which is much lower than that associated with aneurysmal SAH. However, our data suggest that in spite of previous multiple reports of series of patients with NASAH in which there was no associated mortality, some patients with NASAH will die in the acute stage of the disease.

At time of discharge or at the time of the initial follow-up visit, $22 \%$ of patients had either died or required some sort of assistance with daily living that had not been necessary prior to hemorrhage. All other patients were
TABLE 4: Additional complications noted during hospitalization

\begin{tabular}{lc}
\hline Complication & No. of Patients (\%) \\
\hline feeding tube & $7(7.9)$ \\
tracheostomy & $5(5.6)$ \\
deep venous thrombosis & $3(3.4)$ \\
myocardial infarction & $2(2.2)$ \\
seizure & $2(2.2)$ \\
\hline
\end{tabular}

neurologically intact and fully independent. There was a small subset of patients who complained of chronically persistent headaches but who were otherwise at their prehemorrhage baseline. Our outcome data differ sharply from previous outcome reports on patients with NASAH. Only 4 previous studies reported negative outcomes, and in those 4 studies the rates ranged from 2.2 to $12 \%$, $^{2,4,5,9}$

The discrepancy in mortality and morbidity data between our study and the majority of previous series of NASAH patients requires further investigation. Van Gijn and Rinkel have described patients with a particular pattern of NASAH on head CT referred to as "perimesencephalic." The pattern involves accumulation of blood predominantly around the midbrain and has been shown to be associated with a benign clinical course in multiple studies. ${ }^{14-16,22}$ It is possible that our patients had a more serious mortality and morbidity profile than those in previous NASAH case series because they did not represent a perimesencephalic pattern.

Review of all available head CT scans obtained at presentation in our series revealed that only $27 \%$ showed the perimesencephalic pattern of hemorrhage described by Van Gijn and Rinkel. ${ }^{14-16,22}$ It is possible that the term NASAH actually represents multiple disease processes, with one group being the patients with perimesencephalic hemorrhages, and another, potentially more morbid state existing for those patients with NASAH and other hemorrhage patterns.

Six of 95 patients with SAH and a single negative cerebral angiogram were found to have a cerebral aneurysm on subsequent angiograms, resulting in a false negative rate of $6.3 \%$ for initial cerebral angiograms in this study. Our detection rate of $9.4 \%$ for second cerebral angiograms suggests that follow-up imaging in patients with NASAH is indicated.

In our series, multiple modalities of imaging were used for initial and follow-up evaluation. Imaging modalities included cerebral angiograms, CT angiograms, and MR angiogram studies. Nineteen patients did not have follow-up imaging after undergoing cerebral angiography. Of those 19 patients, 4 had bad outcomes. The 3 patients who died did so before long-term follow-up data could be secured. An autopsy was obtained in 1 case and did not reveal any intracranial etiology of hemorrhage. It is feasible, however, that the other 2 deaths could represent patients who had underlying vascular abnormalities that were missed due to lack of follow-up imaging. No follow-up images were obtained in another patient in whom hydrocephalus developed, resulting in a total of 4 patients with bad outcomes who might have had vascu- 


\section{J. Whiting et al.}

lar abnormalities missed because of inadequate followup imaging. Nevertheless, all patients with bad outcomes underwent initial imaging with more than 1 modality and follow-up imaging was performed in some of these patients as well.

\section{Conclusions}

This study suggests that NASAH does not always follow the benign clinical course proposed by previous studies, and that significant morbidity and even mortality can be seen in the context of this disease.

Further investigation is indicated to elucidate possible differences in outcome for patients with NASAH and a perimesencephalic hemorrhage compared with those with other hemorrhage patterns.

\section{Disclaimer}

The authors report no conflict of interest concerning the materials or methods used in this study or the findings specified in this paper.

\section{References}

1. Andrioli GC, Salar G, Rigobello L, Mingrino S: Subarachnoid haemorrhage of unknown aetiology. Acta Neurochir (Wien) 48:217-221, 1979

2. Beguelin C, Seiler R: Subarachnoid hemorrhage with normal cerebral panangiography. Neurosurgery 13:409-411, 1983

3. Brilstra EH, Hop JW, Rinkel GJ: Quality of life after perimesencephalic haemorrhage. J Neurol Neurosurg Psychiatry 63:382-384, 1997

4. Canhao P, Ferro JM, Pinto AN, Melo TP, Campos JG: Perimesencephalic and nonperimesencephalic subarachnoid haemorrhages with negative angiograms. Acta Neurochir (Wien) 132:14-19, 1995

5. Duong H, Melancon D, Tampieri D, Ethier R: The negative angiogram in subarachnoid haemorrhage. Neuroradiology 38:15-19, 1996

6. Giombini S, Bruzzone MG, Pluchino F: Subarachnoid hemorrhage of unexplained cause. Neurosurgery 22:313-316, 1988

7. Hayward RD: Subarachnoid haemorrhage of unknown aetiology. A clinical and radiological study of 51 cases. J Neurol Neurosurg Psychiatry 40:926-931, 1977

8. Hutter BO, Gilsbach JM, Kreitschmann I: Is there a difference in cognitive deficits after aneurysmal subarachnoid haemorrhage and subarachnoid haemorrhage of unknown origin? Acta Neurochir (Wien) 127:129-135, 1994

9. Jain VK, Hedge T, Easwaran RK, Das BS, Reddy GN: Benign subarachnoid haemorrhage (subarachnoid haemorrhage of unknown aetiology). Acta Neurochir (Wien) 86:89-92, 1987

10. Jung JY, Kim YB, Lee JW, Huh SK, Lee KC: Spontaneous subarachnoid haemorrhage with negative initial angiography: a review of 143 cases. J Clin Neurosci 13:1011-1017, 2006

11. Kocak A, Sarac K, Ates O, Cayly SR: Severe cerebral vasospasm caused by non-aneurysmal subarachnoid hemorrhage treatment with transluminal balloon angioplasty. Minim Invasive Neurosurg 50:23-26, 2007

12. Mustonen T, Koivisto T, Vanninen E, Vanninen R, Kuikka JT: Cerebral perfusion heterogeneity and complexity in patients with acute subarachnoid haemorrhage. Nucl Med Commun 27:157-164, 2006

13. Nishioka H, Torner JC, Graf CJ, Kassell NF, Sahs AL, Goettler LC: Cooperative study of intracranial aneurysms and subarachnoid hemorrhage: a long-term prognostic study. III. Subarachnoid hemorrhage of undetermined etiology. Arch Neurol 41:1147-1151, 1984

14. Rinkel GJ, Wijdicks EF, Hasan D, Kienstra GE, Franke CL, Hageman LM, et al: Outcome in patients with subarachnoid haemorrhage and negative angiography according to pattern of haemorrhage on computed tomography. Lancet 338:964968, 1991

15. Rinkel GJ, Wijdicks EF, Vermeulen M, Hageman LM, Tans JT, van Gijn J: Outcome in perimesencephalic (nonaneurysmal) subarachnoid hemorrhage: a follow-up study in 37 patients. Neurology 40:1130-1132, 1990

16. Rinkel GJ, Wijdicks EF, Vermeulen M, Hasan D, Brouwers PJ, van Gijn J: The clinical course of perimesencephalic nonaneurysmal subarachnoid hemorrhage. Ann Neurol 29:463-468, 1991

17. Schwartz TH, Mayer SA: Quadrigeminal variant of perimesencephalic nonaneurysmal subarachnoid hemorrhage. Neurosurgery 46:584-588, 2000

18. Sheehan JM, Cloft H, Kassell NF: Symptomatic delayed arterial spasm following non-aneurysmal perimesencephalic subarachnoid hemorrhage: a case report and review of the literature. Acta Neurochir (Wien) 142:709-712, 2000

19. Suzuki S, Kayama T, Sakurai Y, Ogawa A, Suzuki J: Subarachnoid hemorrhage of unknown cause. Neurosurgery 21:310-313, 1987

20. Tatter SB, Crowell RM, Ogilvy CS: Aneurysmal and microaneurysmal "angiogram-negative" subarachnoid hemorrhage. Neurosurgery 37:48-55, 1995

21. Van Calenbergh F, Plets C, Goffin J, Velghe L: Nonaneurysmal subarachnoid hemorrhage: prevalence of perimesencephalic hemorrhage in a consecutive series. Surg Neurol 39:320-323, 1993

22. van Gijn J, van Dongen KJ, Vermeulen M, Hijdra A: Perimesencephalic hemorrhage: a nonaneurysmal and benign form of subarachnoid hemorrhage. Neurology 35:493-497, 1985

Manuscript submitted January 2, 2009.

Accepted February 16, 2009.

Address correspondence to: Jobyna Whiting, M.D., P.O. Box 100265, University of Florida, Gainesville, Florida 32610. email: whiting@ neurosurgery.ufl.edu. 